Submitted to:

Submitted by:

Prepared by:

Title of Research:

Principal Investigator:

Reporting Period:
National Aeronautics and Space Administration High Energy Astrophysics Division Attention: Dr. Louis Kaluzienski

Code EZ

NASA Headquarters

Washington, DC 20546

The Trustees of Columbia University

in the City of New York

Box 20, Low Memorial Library

New York, New York 10027

Columbia Astrophysics Laboratory

Departments of Astronomy and Physics

Columbia University

538 West $120^{\text {th }}$ Street

New York, New York 10027

"Development of a High-Resolution Liquid Xenon

Imaging Chamber for Gamma-Ray Astronomy"

Elena Aprile

Assistant Professor of Physics

1 May $1989-31$ October 1989
(NASA-CR-185999) A LIQUIO XLNUN IMAGING

TFLFSCOPE FOR $1-30$ MEV TAMMA-RAY

ASTROPHYSICS Semiannual Technical Report, 1

MaY $1989-31$ oct. 1999 (Columbia Univ.)

150

$$
\begin{aligned}
& \text { HA BमN } \\
& \text { IN-39-LR }
\end{aligned}
$$

$239-2$ $15 \%$ 


\section{SEMI-ANNUAL TECHNICAL REPORT}

Submitted to:

Principal Investigator:

Title of Research:

Reporting Period:
National Aeronautics and Space Administration

Attention: Dr. L.J. Kaluzienski

Code EZ

600 Independence Avenue

Washington, D.C. 20546

Elena Aprile

Assistant Professor of Physics

Columbia Astrophysics Laboratory

538 West $120^{\text {th }}$ Street

New York, NY 10027

"Development of a High-Resolution Liquid Xenon Imaging Chamber for Gamma-Ray Astronomy"

1 May $1989-31$ October 1989

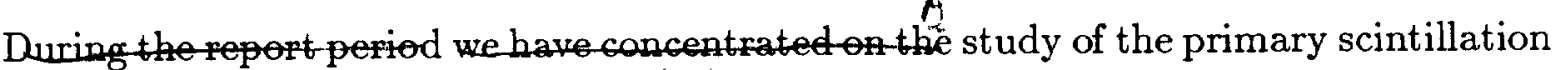

light in liquid xenon excited by ${ }^{241} \mathrm{Am}$ a-particles (see attached paper) and ${ }^{207} \mathrm{Bi}$ internal conversion electrons $\wedge$ The time dependence and the intensity of the light at different field strengths have been measured with a specifically designed chamber, equipped with a $\mathrm{CaF}_{2}$ light transmitting window coupled to a UV sensitive PMT,(Fig. 1). The time correlation between the fast light signal and the charge signal (Eig-2) shows that the scintillation signals produced in liquid xenon by ionizing particles provides an ideal trigger in a Time Projection typg LXe detector aiming at full imaging of complex $\not$-ray events.

also started Monte Carlo calculations to establish the performance of a LXe imaging telescope for high energy $\gamma$-rays. 


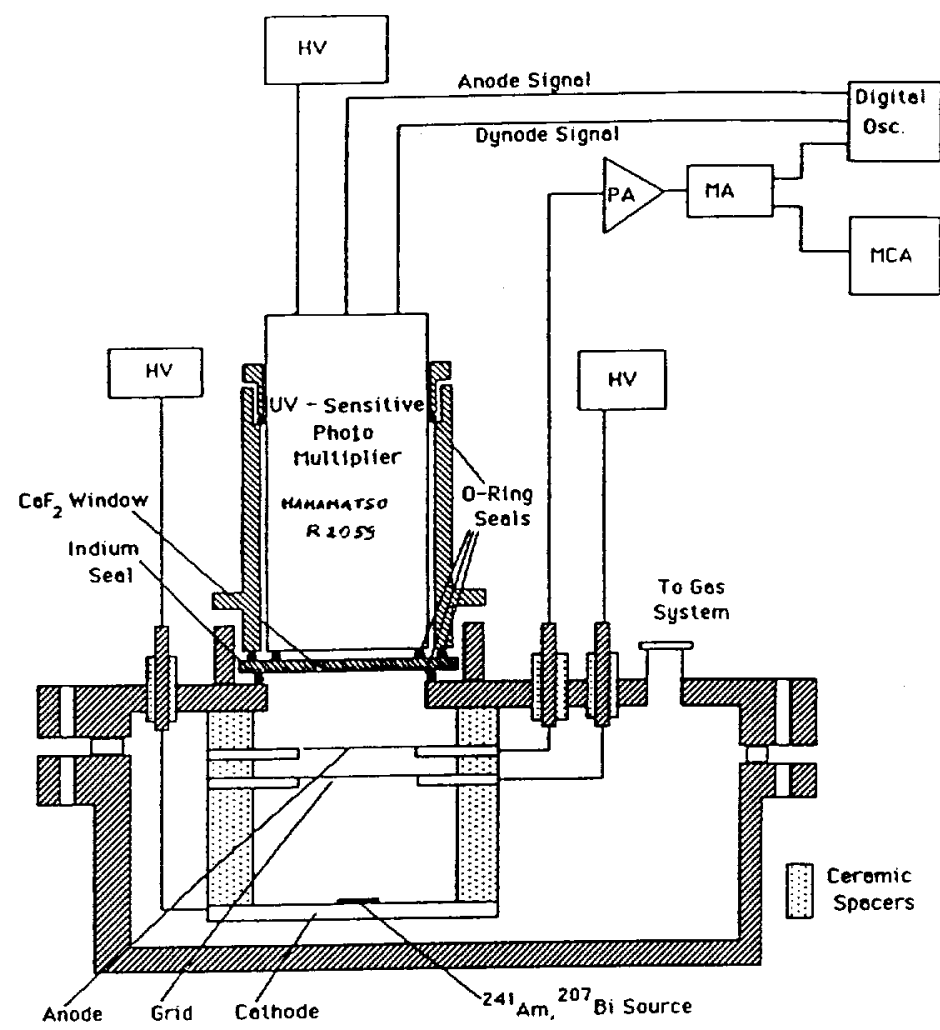

Figure 1: Liquid Xenon Scintillation Chamber

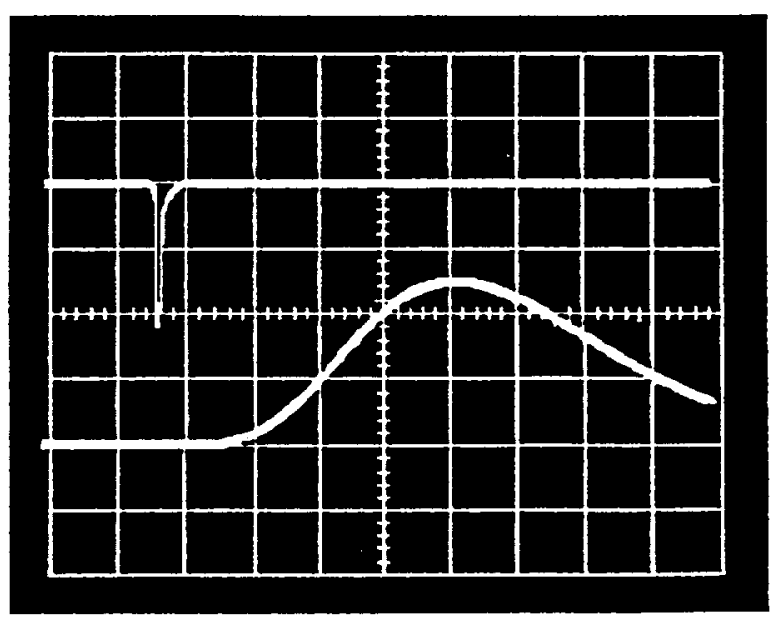

Figure 2: ${ }^{241} \mathrm{Am}$ alpha particle ionization signal triggered by the scintillatin light ( $500 \mathrm{mV} /$ div., $2 \mu \mathrm{s} /$ div.). 


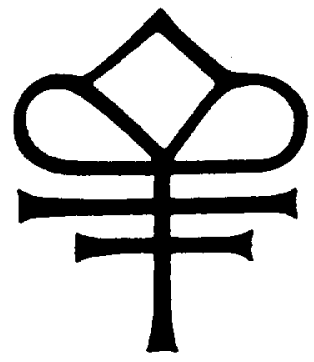

A Liquid Xenon Imaging Telescope for 1-30 MeV Gamma-Ray Astrophysics

Elena Aprile, Reshmi Mukherjee, and Masayo Suzuki

Columbia Astrophysics Laboratory

Columbia University, New York, NY 10027

To appear in: Proceedings of the SPIE 33rd Annual International Symposium on Optical \& Optoelectronic Applied Science and Engineering

San Diego, 6 - 11 August 1989

\section{COLUMBIA UNIVERSITY}

DEPARTMENTS OF

PHYSICS and ASTRONOMY

NEW YORK, NEW YORK 10027

Columbia Astrophysics Laboratory Contribution no. 388 


\title{
A Liquid Xenon Imaging Telescope for 1-30 MeV Gamma-Ray Astrophysics
}

\author{
Elena Aprile, Reshmi Mukherjee, and Masayo Suzuki \\ Columbia Astrophysics Laboratory \\ Columbia University, New York, NY 10027
}

\begin{abstract}
We discuss the application of a high resolution liquid xenon imaging chamber as a $\gamma$-ray telescope for medium energy astrophysics. The chamber, operated in the Time Projection Mode, will be capable to image any ionizing event occurring within its sensitive volume. Gamma-ray events with multiple Compton interactions will be identified as such, thus significantly enhancing the detection efficiency over a wide energy range. This represents the main advantage of the proposed liquid xenon instrument over more conventional double scatter Compton telescopes, in which stringent topological constraints limit considerably the acceptance. In the Compton regime, the excellent energy and position resolution of the detector, on the order of $3 \%$ FWHM at $1 \mathrm{MeV}$ and of $250 \mu \mathrm{m}$ RMS, respectively, will result in a $1 \sigma$ angular resolution of $0.5^{\circ}-0.3^{\circ}$ for $\gamma$-ray energies of $1-20 \mathrm{MeV}$. The ambiguity in source location that characterizes the Compton measurement can be removed for $\gamma$-ray events of high enough energy to allow the reconstruction of the direction of the Compton electron. In the pair production regime, the incoming $\gamma$-ray direction can also be uniquely determined from the reconstructed openining angle of the electron-positron pair. An effective discrimination against charged and neutral background events is a direct consequence of the detector imaging capability. The versatility of the liquid xenon telescope and its unique characteristics will allow a large variety of $\gamma$-ray emitting sources to be explored with high sensitivity.
\end{abstract}

\section{INTRODUCTION}

Observations of the gamma-ray sky in the low and medium energy range below about $30 \mathrm{MeV}$ promise to provide a wealth of information on the nature of the source or region in which the gamma-rays are produced. The difficult experimental problems encountered in this energy region have resulted in a slower development than in other regions of the electromagnetic spectrum. Even though considerable progress has been achieved during the last few years and a wealth of new information is expected from the four instruments planned for the Gamma Ray Observatory (GRO) mission, it is clear that improved experimental techniques are needed for future gamma-ray observations. The application of rare gas liquids, such as xenon, promise major advances in detector efficiency, imaging and background reduction capabilities as well as detector energy and spatial resolution.

Among the properties which make a liquid xenon imaging chamber especially suited for gamma-ray astronomy are:

1. High detection efficiency due to the high density $\left(\rho=3.06 \mathrm{~g} / \mathrm{cm}^{3}\right)$ and high atomic number $(Z=54)$.

2. Excellent energy resolution, ultimately similar to that of germanium.

3. Excellent spatial resolution, ultimately a few tens of $\mu \mathrm{m}$.

4. Excellent scintillation efficiency, comparable to that of $\mathrm{NaI}(\mathrm{Tl})$ and with a fast component, ideal for event triggering.

5. Three-dimensional track reconstruction which allows bubble chamber like images of multiple gamma-ray interactions.

6. Excellent background rejection capability due to track imaging and to pulse shape discrimination of the scintillation signal.

7. Short radiation length $\left(L_{\mathrm{rad}}=2.6 \mathrm{~cm}\right)$, ideal for a compact calorimeter. 
The combination of these properties in one detector is especially desirable if one wants to efficiently and unambiguously identify the location in the sky of unknown gamma-ray emitting objects. Compared with conventional Compton telescopes which aim at this goal, the proposed liquid xenon imaging telescope offers considerable increase in detector efficiency, angular resolution and background rejection capability.

The imaging capability of the instrument will allow to visualize complex events with multiple gammaray interactions, initiated by either Compton scattering or pair production. As a result of this imaging, efficient background event rejection is also achieved. The accuracy in the determination of the incident gamma-ray direction depends on the gamma-ray energy. For gamma-rays in the few $\mathrm{MeV}$ range, whose energy is totally absorbed after an initial Compton interaction, the liquid xenon chamber can be used as a conventional Compton telescope, with the difference that the interaction points of the incoming and scattered gamma-ray are identified in a single detector rather than in two separate ones, at a fixed distance. The gamma-ray scattering angle and its energy will be inferred from the measurement of the coordinates of the two interaction points and of the energies deposited in the liquid xenon. The known scattering angle then restricts the direction of the incoming photon to a cone with its axis along the direction of the scattered photon. The projection of the cone onto the celestial sphere represents a ring, of radius equal to the scattering angle and width determined by the precision of the energy measurement. The source of emission is then identified as the point of intersection of all such rings. With a spatial resolution of $250 \mu \mathrm{m}$ and an energy resolution of $3 \%$ FWHM at $1 \mathrm{MeV}$, the overall angular resolution of the liquid xenon Compton telescope is estimated to be on the order of $0.5^{\circ}$ at $1 \mathrm{MeV}$ and better at higher energies.

For higher energy gamma-rays which produce Compton electrons and positron-electron pairs of sufficient energy to permit their imaging in the liquid xenon chamber, the localization of the source on the celestial sphere can be uniquely determined.

The use of liquid xenon in a practical large size instrument with high spectral and spatial resolution has been mostly limited up to now by the technical problem of achieving and mantaining the high level $(<1$ part per billion of electronegative substances) of purity required for drifting ionization electrons over a large distance in the liquid, without appreciable loss in the detectable signal. Our recent experimental results in this area show that we can reliably purify xenon down to the ppb level of oxygen equivalent even for large quantities of liquid.

\section{INSTRUMENT DESCRIPTION AND PROPERTIES}

The proposed $\gamma$-ray telescope is a fully active liquid xenon ionization chamber, operated as a Time Projection Chamber (TPC). The device is continuously sensitive to any jonizing event. Gamma-rays interacting within the sensitive volume produce secondary particles by photoabsorption, Compton scattering, or pair production. These charged particles will dissipate their energy by ionization and excitation of the liquid, creating a large number of electron-ion pairs along their trajectory, as well as scintillation photons. Under the influence of an external electric field, the ionization electrons which escape recombination with the positive ions and which are not trapped by impurities will drift from their point of creation towards a collection electrode structure. The total energy and the spatial distribution of an ionizing event can be measured from the induced charge signals on the collector. The chamber thus has the ability to produce bubble chamber like images of the $\gamma$-ray events under investigation. Since the full event is recorded, no topological constraints limit the acceptance, enormously increasing the detection efficiency. To obtain similar efficiency with the more common techniques used in $\gamma$-ray astronomy, much larger detectors or longer observation times would be required.

To implement the imaging capability of the liquid xenon TPC, different readout schemes can be adopted. The two most commonly used are multi-wire structures, in which the drifting electrons are passed from one sensing plane to the next determining one coordinate of the electron cloud at a time, or segmented anodes, with an interwoven pattern of strips in both directions printed on one plate. We are presently testing a segmented anode of the type used by Mahler et al. (1983) for a liquid argon TPC, but with much finer segmentation. A spacing between the sensitive elements of the order of $0.5 \mathrm{~mm}$ appears feasible, and should yield $\sim 250 \mu \mathrm{m}$ RMS spatial resolution.

To minimize charge losses in the detectable signal, the liquid filling the chamber has to be free from electronegative impurities which attach free electrons, the applied electric field has to be sufficinetly strong to reduce the electron-ion recombination process, and the transparency of the shielding grid has to be 
maximized. We have extensively studied all these problems and feel confident to proceed with the realization of a $\gamma$-ray instrument.

A schematic drawing of the liquid xenon telescope under consideration is shown in Fig 1 . The active volume is about $3 \times 10^{4} \mathrm{~cm}^{3}$ and the sensitive area is $1600 \mathrm{~cm}^{2}$. The depth of $26 \mathrm{~cm}$ of liquid xenon corresponds to ten radiation lengths and should insure a high conversion efficiency for high energy $\gamma$-rays.

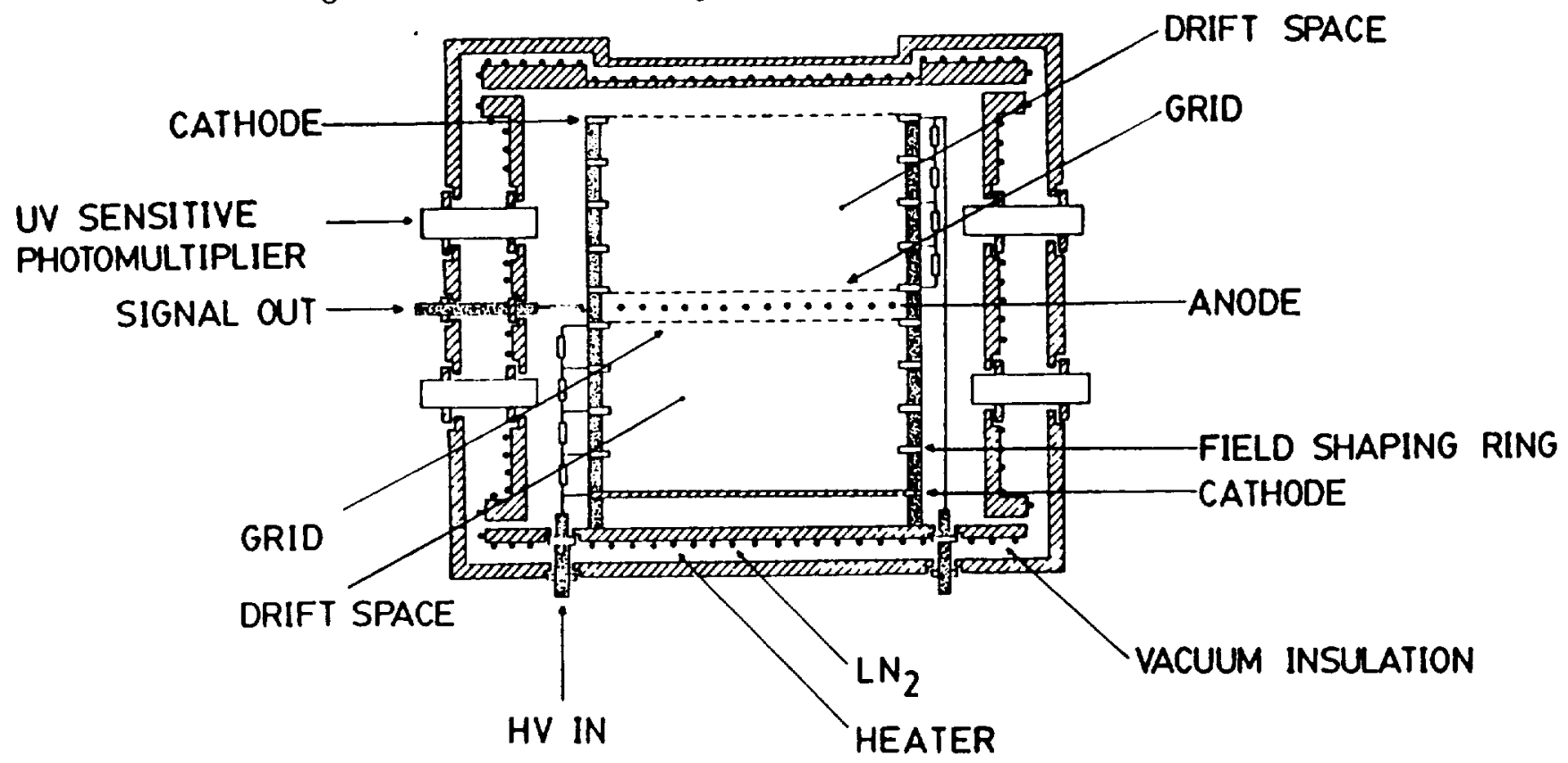

Figure 1. Schematic of the liquid xenon imaging chamber.

The total active volume of the detector is subdivided into 2 drift regions, each $13 \mathrm{~cm}$ long. Ionization electrons produced by an ionizing event can drift from both sides towards a central readout electrode structure. The uniformity of the electric drift field is insured by a sequence of field shaping rings. High voltage is applied to the cathode planes made of thin mesh to minimize the interaction of $\gamma$-rays with passive materials. A liquid nitrogen heat exchanger can be used to maintain the required liquid xenon operating temperature.

The UV sensitive photomultipliers, two for each drift region, will detect the primary scintillation light to be used for triggering. The ionization signals induced on each sensing element of the collector, will be fed to low noise charge-sensitive preamplifiers. These will be mounted directly on the chamber, as close as possible to the readout plane to avoid a large cable capacitance, and to take advantage of the reduced noise levels of cooled field effect transistors (FETs). The signals from the preamplifiers will be multiplexed into wave form digitizers. These will be continuously active to provide a uniform sampling of the collected charge in the $X$ - and $Y$-directions. The degree of multiplexing depends on event complexity and total event rate.

\subsection{Energy Resolution}

The energy resolution of a liquid xenon ionization chamber is theoretically limited by statistical fluctuations in the total number of charge carriers produced. For liquid xenon, the small values of the calculated Fano factor $(F=0.04)$ and of the measured average energy to produce an ion pair $(\mathrm{W}=15.6 \mathrm{eV})$ yield a theoretical energy resolution $\Delta E / E=2.35\left[(F W / E)^{1 / 2}\right]=0.2 \%(2 \mathrm{keV})$ for an electron energy of $E=1$ $\mathrm{MeV}$ (Doke et al. 1976).

Different factors contribute however to a degradation of this ultimate energy resolution: they include charge loss mechanisms such as attachment and recombination, electronic noise and geometrical effects. 
We have extensively studied the influence of these different factors on the spectral performance of noble liquid detectors, with systematic measurements in both liquid argon and liquid xenon. The electric field dependence of the charge yield and of the energy resolution of electrons, $\gamma$-rays and alpha particles has been measured (Aprile et al. 1987; Aprile et al. 1988; Aprile and Suzuki 1989). A typical energy spectrum obtained with a liquid xenon gridded chamber, irradiated by an internal ${ }^{207} \mathrm{Bi}$ radioactive source is shown in Fig. 2. The dominant $570 \mathrm{keV} \gamma$-ray line is observed with an intrinsic resolution of $34 \mathrm{keV} \mathrm{FWHM}$. A dependence as $E^{-1 / 2}$ can be assumed for the energy resolution at higher energies.

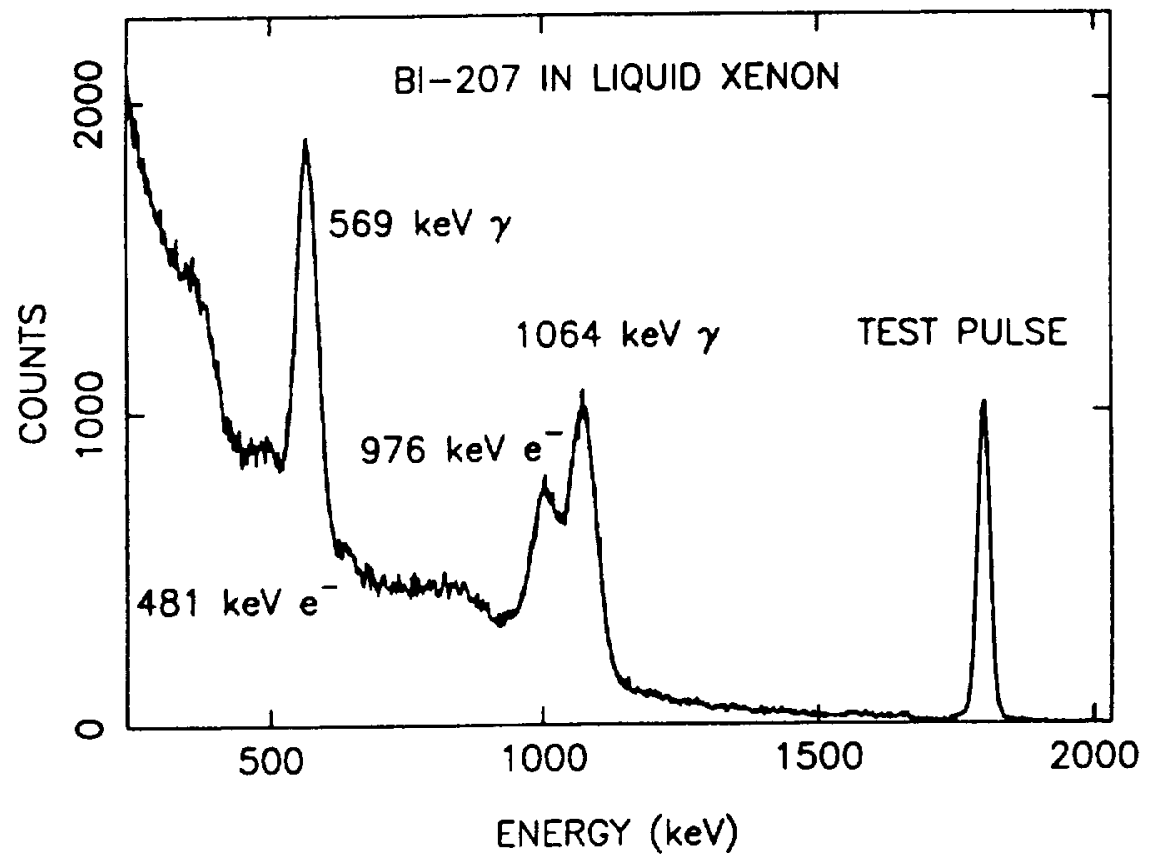

Figure 2. Energy spectrum of ${ }^{207} \mathrm{Bi}$ in liquid xenon.

Our experimental values for the energy resolution in liquid argon and xenon are the best achieved so far, but still far from the theoretical limits based on the Fano factor. To explain this discrepancy, we have advanced the hypothesis of a stronger recombination rate on the tracks of low energy delta-rays, abundantly produced by the primary ionizing particle. Recombination fluctuations in the number of collected electrons from delta-tracks thus dominate the observed energy resolution, producing much larger fluctuations than those expected from the Fano factor. This hypothesis, consistent with our results, provides for the first time a good explanation of the limited energy resolution results obtained in high density rare gas chambers under typical drift fields.

We are currently continuing our studies with small volume liquid xenon detectors to better understand the separate effects of recombination and electron attachment and therefore improve the spectral performance. Although, under practical operating conditions, it seems difficult to reach the ultimate energy resolution, an improvement to the $3 \%$ level at $1 \mathrm{MeV} \gamma$-ray energy, is feasible.

\subsection{Spatial Resolution}

Spatial resolution is the most important detector parameter for event imaging. The diffusion of the drifting electrons in the applied field, multiple scattering and the production of energetic delta-rays are the dominant limitations for position resolution in a liquid ionization device. Technical limitations include electric field distortions and practical constraints on the spacing between the wires or strips of the readout electrodes.

The RMS spread of the center of gravity of a cloud of electrons, due to the diffusion process is given by $\left(2 D t_{d} / N\right)^{1 / 2}$ where $D$ is the diffusion coefficient, $t_{d}$ is the electron drift time and $N$ is the number of electrons in the cloud. The diffusion coefficient in liquid xenon is several times larger than that in liquid 
argon, but still 50 times smaller than in xenon gas at one atmosphere (Shibamura et al. 1979). Assuming $D=65 \mathrm{~cm}^{2} / \mathrm{sec}$, a drift velocity of $2.3 \times 10^{5} \mathrm{~cm} / \mathrm{sec}$ under an electric field of $3 \mathrm{kV} / \mathrm{cm}$ and $N=6.4 \times 10^{4}$ electrons for $1 \mathrm{MeV}$ ionizing radiation, we estimate a lateral diffusion of the center of gravity of $3 \mu \mathrm{m}$ for a drift path of $10 \mathrm{~cm}$.

The resolution on the $X$ and $Y$ coordinates, orthogonal to the drift axis, is therefore mainly determined by the spacing between the detector read-out wires or sensing elements, and thus by system cost considerations and by questions of mechanical stability. In the ionization mode, spatial resolution on the order of 20 $\mu \mathrm{m}$ RMS have been achieved with small liquid xenon and liquid argon chambers (Muller et al., 1971; Deiters et al., 1981). With a large size detector a spatial resolution of a few hundred $\mu \mathrm{m}$ should be achievable with a more realistic wire spacing of $500 \mu \mathrm{m}$.

Much better resolution, on the order of the diffusion limited value, is expected on the $Z$ coordinate, which is derived from drift time measurements. This is due to the property that the electron drift velocity in liquid xenon is almost constant for electric fields higher than $3 \mathrm{kV} / \mathrm{cm}$ (Miller et al. 1968).

\subsection{Scintillation Light}

The primary scintillation light, abundantly produced by a charged particle in liquid xenon, represents an ideal trigger source for the TPC. The scintillation efficiency of xenon is very high, comparable to that of $\mathrm{NaI}(\mathrm{Tl})$ but with shorter decay times. The wavelengths of the scintillation photons are centered around $170 \mathrm{~nm}$, which requires exit windows of $\mathrm{CaF}_{2}, \mathrm{BaF}_{2}$, or UV grade sapphire. The time dependence of the scintillation light yield on the applied electric field has been measured by Kubota et al. $(1979,1982)$. Under the condition of minimal recombination, achieved with a strong electric field, the light pulse consists of a fast component with a decay time of about $2.2 \mathrm{nsec}$ and a slow component of about $27 \mathrm{nsec}$, with an intensity ratio of about 6 in favour of the slow component.

We have recently measured the light signal, the charge signal and their correlation in liquid xenon excited by ${ }^{241} \mathrm{Am}$ alpha particles. The ionization chamber used for these measurements was equipped with a two inch diameter UV sensitive PMT coupled to the sensitive volume with a $\mathrm{CaF}_{2}$ window. It has worked reliably at liquid xenon temperature, providing very encouraging results. Figure 3(a) shows the dynode pulse of the photomultiplier, corresponding to the total energy of the alpha particles, triggered with the anode pulse of the photomultiplier. Figure 3(b) shows instead the ionization signal of the alpha particles, as detected by the charge sensitive preamplifier, triggered with the anode pulse of the photomultiplier. The applied drift field was $5 \mathrm{kV} / \mathrm{cm}$. Tests of the same chamber irradiated with an electron source are currently underway.

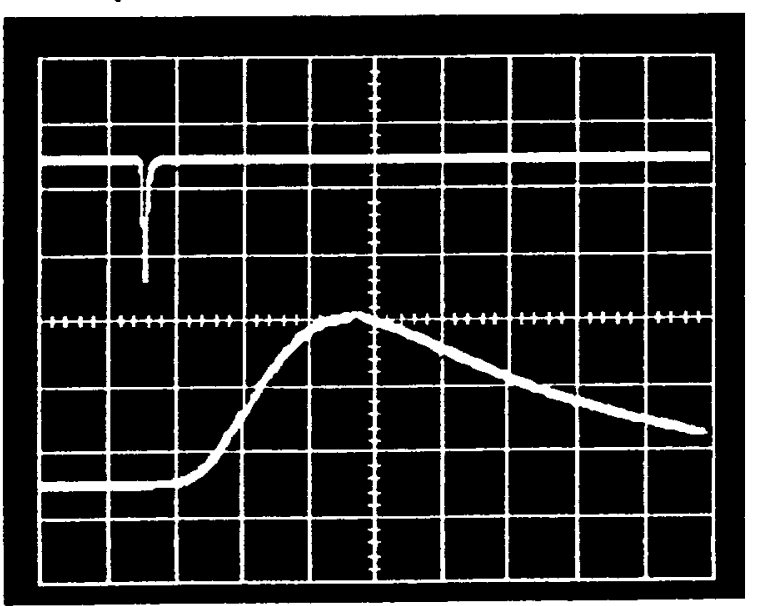

Figure 3(a). Scintillation light of ${ }^{241} \mathrm{Am}$ alpha particles in liquid xenon (upper trace: $500 \mathrm{mV} /$ div., $500 \mathrm{~ns} /$ div.), and its integrated dynode pulse (lower trace: $1 \mathrm{~V} /$ div., $500 \mathrm{~ns} /$ div.).

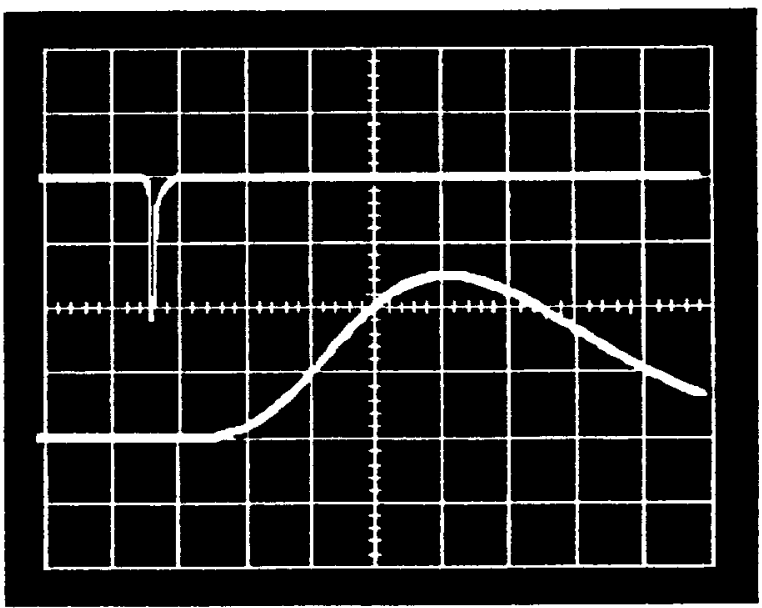

Figure 3(b). ${ }^{241}$ Am alpha particles ionization signal triggered by the scintillation light $(500 \mathrm{mV} /$ div., $2 \mu \mathrm{s} /$ div.). 


\section{EXPECTED INSTRUMENT PERFORMANCE}

\subsection{Background Rejection}

A liquid xenon Time Projection Chamber images all ionizing events occurring within its sensitive volume. This imaging capability can be used not only to analyze the topology of the events under investigation, but also to reject background, reducing the requirement for a massive anticoincidence shield of the type that is required for germanium and sodium iodide detectors. High energy charged particles entering the chamber are unambiguosly identified and therefore may be removed since the initial part of the track is located at the very edge of the sensitive volume. Low energy neutral background reaching the detector will predominantly interact close to the edges of the chamber. A strong background of this sort can be easily detected in the spatial distribution of the interaction points. These distributions also indicate the necessary fiducial volume cuts for background suppression. Pulse shape discrimination of the measured scintillation pulses represents an additional means for identifying such background. The scintillation light in liquid xenon in fact depends strongly on the ionization density of the event. This has been demonstrated with extensive measurements by Kubota et al. (1980). Neutron induced events for example can be easily discriminated against, since the scintillation signal produced by a proton or an alpha particle is quite different from that produced by an electron.

\subsection{Angular Resolution in the Compton and Pair Regime}

The most important benefit from imaging is, however, the possibility of reconstructing the complete geometry of each gamma-ray event, and to identify its topology. Since the full event is registered, multiple Compton interactions can be recognized as such. To evaluate the performance of the proposed liquid xenon chamber as an imaging $\gamma$-ray telescope, we have carried out preliminary Monte Carlo calculations, using the "Electron Gamma Shower" (EGS4) computer program (Ford and Nelson,1978), which simulates all possible interaction processes initiated by incident $\gamma$-rays. Figure 4 shows the number of $\gamma$-ray events which interact in the liquid xenon with a single Compton scattering as compared to those which undergo two or more Compton interactions. In both case we have required that the total energy of the incident $\gamma$-ray is contained within the detector. At high energy the number of multiple interaction events is not negligible. These events which have a high probablility to be rejected in a conventional double scatter Compton telescope, carry as much information as single Compton events, if all the interaction points and the deposited energy in each point are measured. The imaging capability of the TPC, thus, enhances the detector efficiency by correctly identifying these events. At low energies, the probability for multiple Compton events is reduced, but the $\gamma$-ray can be easily photoabsorbed shortly after the Compton scattering. In a conventional double scatter Compton telescope these events will be rejected, since the two interactions occur in the same part of the detector.

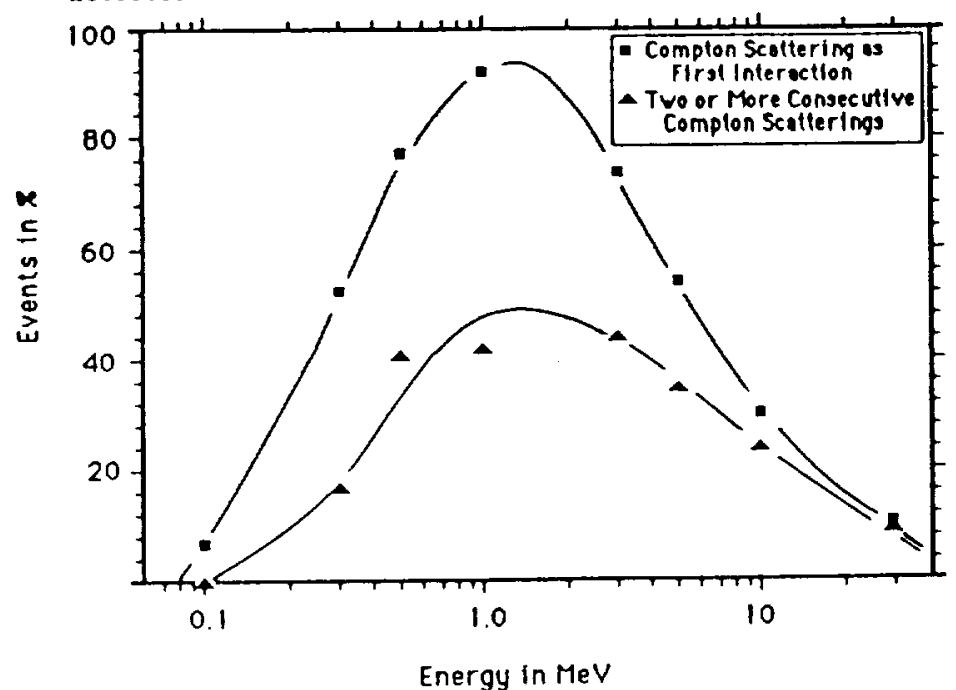

Figure 4. Expected number of $\gamma$-ray events with a single Compton scattering ( $\boldsymbol{(})$ and with two or more consecutive Compton scatterings (A). 
In the 1-30 MeV medium energy range, Compton scattering and pair production are the dominant gamma-ray interaction processes, as shown in Fig. 5. Simple estimates of the expected angular resolution of the liquid xenon telescope operated in the Compton and pair production regimes will be made for typical event topologies generated by $1 \mathrm{MeV}$ and $20 \mathrm{MeV}$ incident gamma-rays.

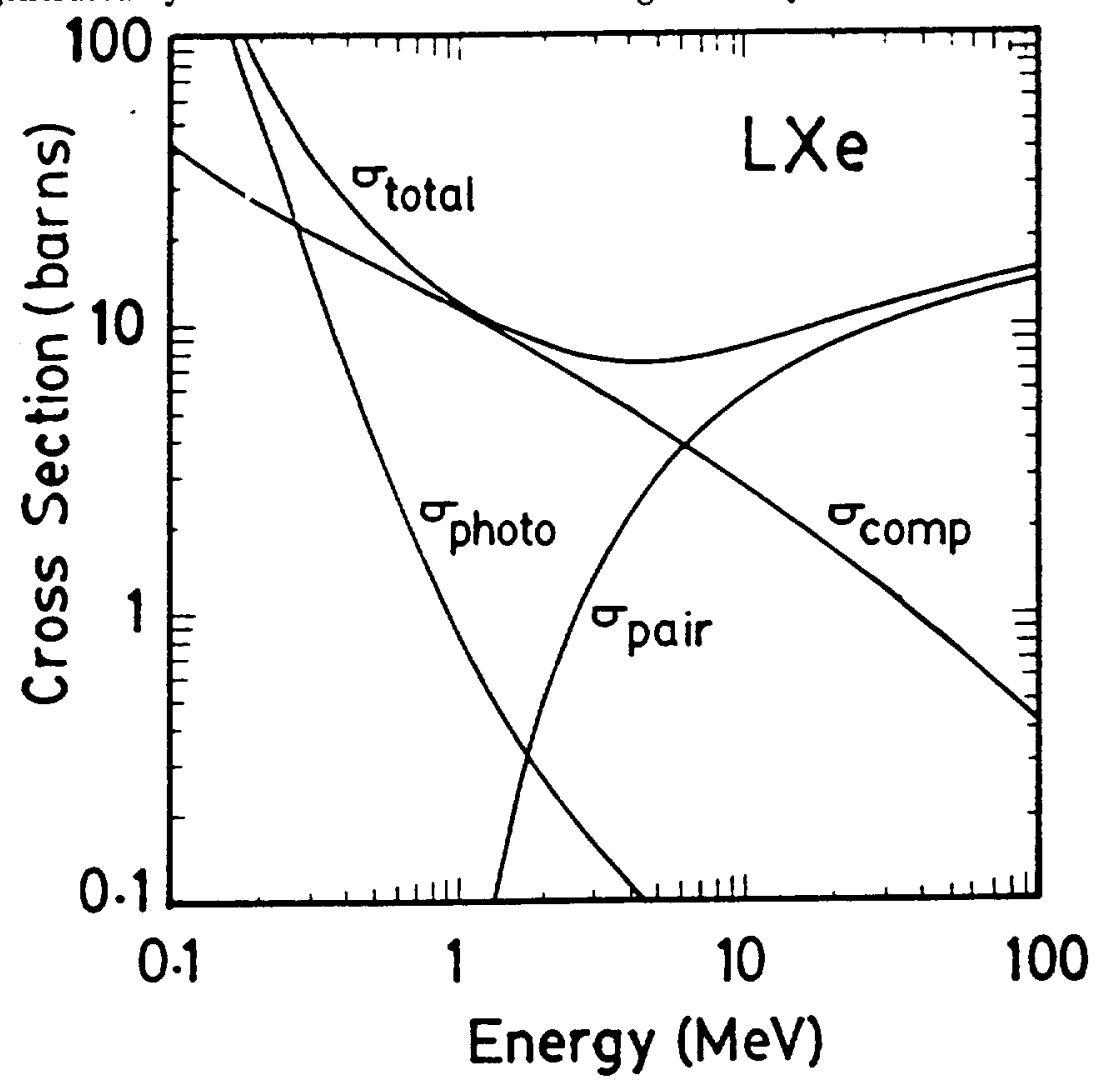

Figure 5. Total photon absorption cross section for liquid xenon.

In liquid xenon, low energy electrons loose their energy over a distance of a few millimeters. The short track lengths are further complicated by multiple scattering. With the assumed spatial resolution of $250 \mu \mathrm{m}$ of the proposed instrument, we do not expect to be able to reconstruct the direction of low energy Compton electrons. To estimate the angular resolution for $1 \mathrm{MeV}$ and $20 \mathrm{MeV}$ gamma-rays, we therefore simply assume the same measurement principle as that of conventional double scatter Compton telescopes. The difference is that the gamma-ray interactions are measured in a single detector rather than in two different detectors separated by a fixed distance.

In the liquid xenon chamber operated as a Compton telescope the incident gamma-ray with energy $E_{\gamma}$ is identified by two successive interactions in the active volume: the first one is a Compton scattering, in which the primary photon tranfers the energy $E_{e}^{\prime}$ to an electron and scatters by an angle $\phi$ (see Fig. 6). The scattered photon with reduced energy $E^{\prime}$ is identified in a second interaction, where it looses an energy $E_{e}^{\prime \prime}$, which coincides with $E_{\gamma}^{\prime}$ if it is totally absorbed. From the measured values of $E_{e}^{\prime}$ and $E_{e}^{\prime \prime}$ one can determine the energy of the primary photon and its scattering angle according to the relations:

$$
E_{\gamma}=E_{e}^{\prime}+E_{e}^{\prime \prime} \text { and } \cos \phi=1-m c^{2}\left(\frac{1}{E_{e}^{\prime \prime}}-\frac{1}{\left(E_{e}^{\prime}+E_{e}^{\prime \prime}\right)}\right)
$$

where $\mathrm{mc}^{2}$ is the rest energy of an electron. If, in addition, the coordinates of both interaction points are measured, the arrival direction of the incident gamma-ray lies on the surface of a cone of half opening angle $\phi$, around the direction of the scattered gamma-ray. A source in the sky may be identified as the intersection point of all the event circles, defined as the projections onto the celestial sphere of the cones. The width of the rings depends on the precision of the energy measurement. 


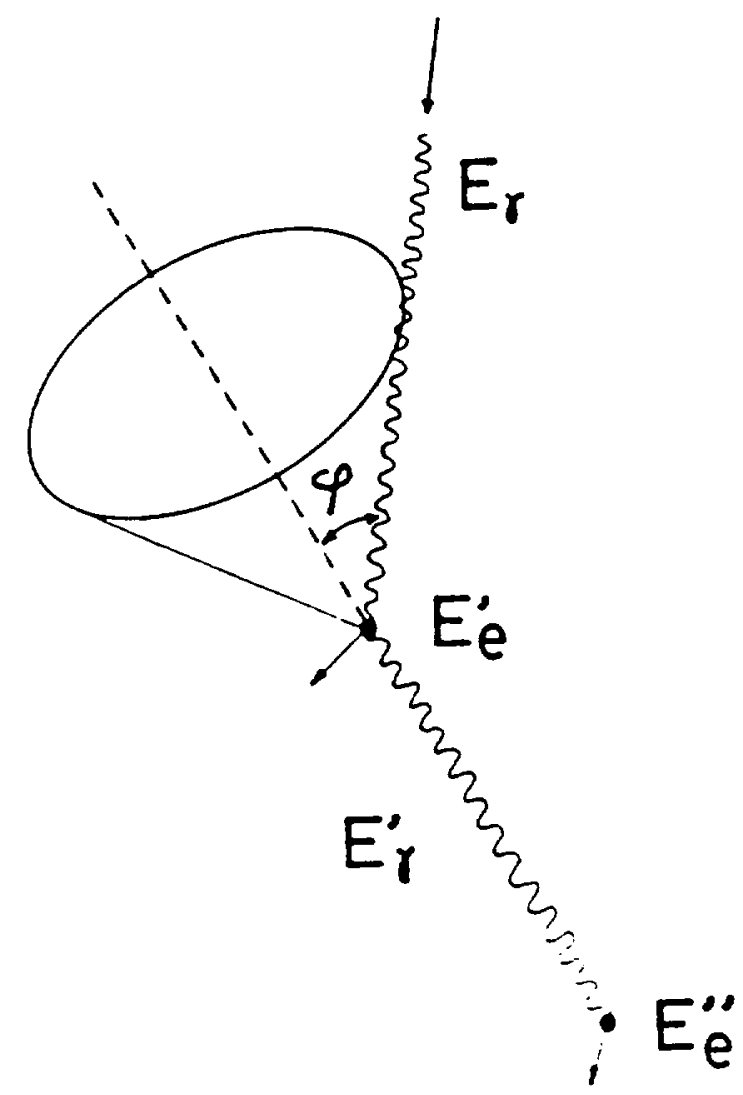

Figure 6. Principle of the Compton measurement.

The ambiguity of this measurement in identifying the true source of gamma-ray emission can be completely removed if the direction of the Compton electron can also be accurately measured.

The probability that a gamma-ray incident upon the liquid xenon undergoes a Compton scattering varies between approximatley $90 \%$ at $1 \mathrm{MeV}$ and $20 \%$ at $20 \mathrm{MeV}$. The total cross section at $1 \mathrm{MeV}$ is 12 barns/atom. The corresponding photon attenuation length $\lambda$ is $7.1 \mathrm{~cm}$, as shown in Fig. 7. As the angular distribution of the scattered gamma-rays peaks at $\phi=25^{\circ}$ for $E_{\gamma}=1 \mathrm{MeV}$, the following configuration represents the most typical case: $E_{\gamma}^{\prime}=0.85 \mathrm{MeV}, \phi=25^{\circ}, E_{e}^{\prime}=0.15 \mathrm{MeV}$ and $\theta=57^{\circ}$, with $\theta$ the electron scattering angle. The scattered gamma ray with $0.85 \mathrm{MeV}$ of energy will interact with liquid xenon within $5.5 \mathrm{~cm}$ of the first interaction point with a probability of $63 \%$. If the total energy of the incident gammaray is contained within the sensitive volume, $E_{\gamma}^{\prime}=E_{e}^{\prime \prime}=0.85 \mathrm{MeV}$. The overall angular resolution of the telescope depends both on the accuracy in the location determination of the two interaction points and on the accuracy of the energy measurements. The first contribution is determined by the spatial resolution of the detector. Assuming a spatial resolution on both $X$ - and $Y$-coordinates of $\Delta X=\Delta Y=250 \mu \mathrm{m}$, and $\lambda=5.5 \mathrm{~cm}$ as the distance between the two points, we estimate an angular resolution of $0.26^{\circ}$ for the arrival direction of the gamma-ray (cone's axis). The second contribution is determined by the energy resolution of the detector, which transforms into an uncertainty in the photon scattering angle (cone's half opening angle) according to the relation:

$$
\Delta \phi=\frac{180 \mathrm{mc}^{2}}{\pi \sin \theta}\left[\frac{\left(\Delta E_{e}^{\prime}\right)^{2}}{E_{\gamma}^{4}}+\left(\Delta E_{c}^{\prime \prime}\right)^{2}\left(\frac{1}{\left(E_{e}^{\prime \prime}\right)^{2}}-\frac{1}{E_{\gamma}^{2}}\right)^{2}\right]^{\frac{1}{2}}
$$

where $\Delta E_{e}^{\prime}$ and $\Delta E_{e}^{\prime \prime}$ are the $1 \sigma$ uncertainties on $E_{e}^{\prime}$ and $E_{e}^{\prime \prime}$, respectively. Assuming an energy resolution of $3 \%$ FWHM at $1 \mathrm{MeV}$ and a scaling law for $\Delta E$ as $\frac{\Delta E}{E}=\frac{1.28}{\sqrt{E}}(\%)$ with $E$ in unit of $\mathrm{MeV}$, we obtain $\Delta \phi=0.46^{\circ}$ for a $25^{\circ}$ scattering angle. The overall angular resolution is the sum in quadrature of the two contributions, or $0.53^{\circ}$. This value is already competitive with the angular resolution of existing Compton telescopes. 


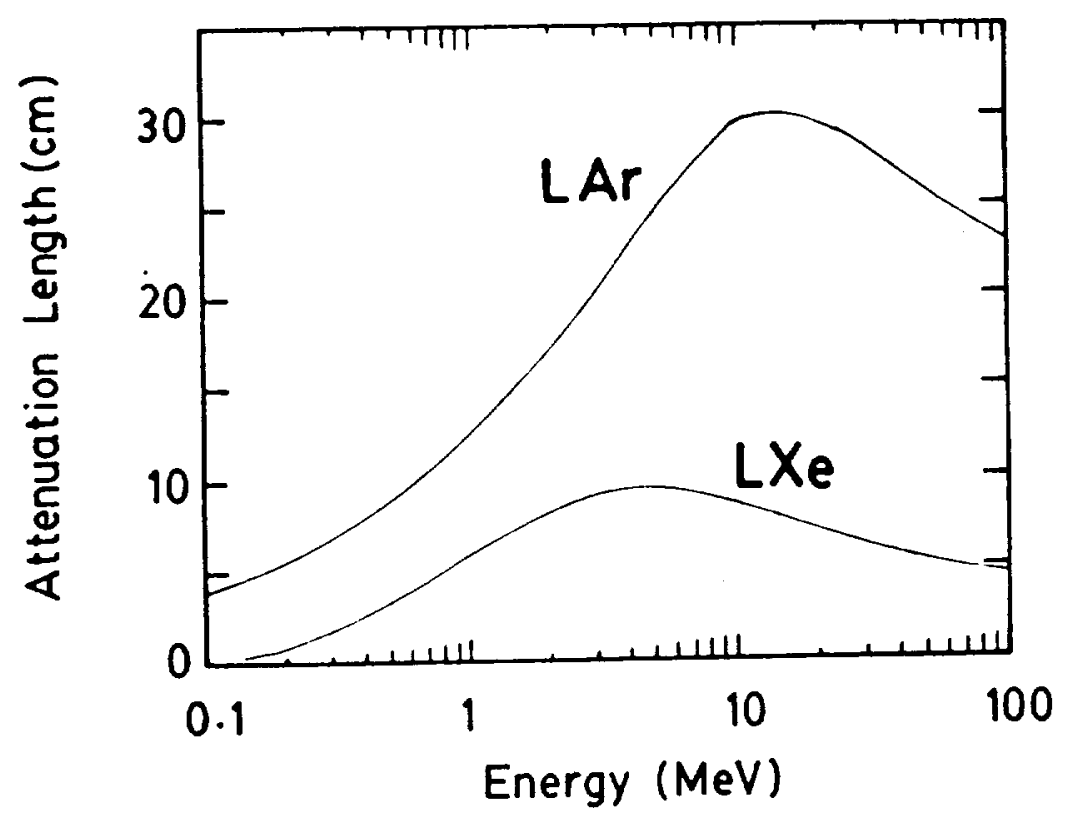

Figure 7. Photon attenuation length in liquid argon and xenon.

At $20 \mathrm{MeV}$ there is still about $20 \%$ probability that Compton scattering takes place as the first interaction instead of pair production. Since the angular distribution of the scattered gamma-ray peaks at $5^{\circ}$, the following event configuration represents the most typical case: $E_{\gamma}^{\prime}=18 \mathrm{MeV}, \phi=5^{\circ}, E_{c}^{\prime}=2 \mathrm{MeV}$ and $\theta=12^{\delta}$. The scattered gamma-ray will then interact via pair production with an $80 \%$ probability. Assuming that the total energy of this second interaction is contained within the detector, we estimate an overall angular resolution of $\sim 0.3^{\circ}$ for the incident gamma-ray direction, using the measurements of the energy and of the interaction points as described above.

For those Compton events with the scattered electron of high enough energy to be accurately imaged, a unique determination of the source location becomes possible as previously pointed out.

At higher gamma-ray energies the probability for Compton scattering decreases rapidly and pair production takes over. The imaging capability of the proposed telescope will allow one to identify the more complex event topologies with multiple interactions expected at high energy. In particular, if the first interaction of the incident gamma-ray in liquid xenon is the production of a pair, the gamma-ray direction can be uniquely determined with a resolution of the order or one degree, using track imaging. As an example we discuss the interaction of a $20 \mathrm{MeV}$ gamma-ray incident upon the liquid xenon telescope.

At $20 \mathrm{MeV}$ the total cross section in liquid xenon is $10 \mathrm{barns} /$ atom, and the corresponding photon attenuation length, $\lambda$, is $8.5 \mathrm{~cm}$. The most probable interaction of a gamma ray of this energy incident upon liquid xenon is pair production. The total energy of the electron and positron generated will be $8.98 \mathrm{MeV}$, with an equal partition of the energy being the most probable case. The average opening angle of the pair is very small, of order $\mathrm{mc}^{2} / E_{\gamma} \simeq 3^{\circ}$. The range of a $9 \mathrm{MeV}$ electron in liquid xenon is on the order of a few centimeters, and the trajectory is distorted by multiple scattering. However, since the electron and positron will loose most of their energy at the end of their path, the initial part of their tracks should be fairly straight. As a result, their imaging is possible and the direction of the incident gamma-ray can be uniquely determined with a precision better than half the opening angle of the pair.

\subsection{Detection Efficiency}

The most important advantage of the proposed telescope over conventional ones is the significantly enhanced detection efficiency over a wide energy range. Figure 8 shows the detection efficiency for vertical incidence as a function of $\gamma$-ray energy, from Monte Carlo calculations. We have assumed a $26 \mathrm{~cm}$ deep active volume and $1600 \mathrm{~cm}^{2}$ geometrical area. Two different curves are shown. The line through the data points marked as $\Delta$ represents $\gamma$-ray events with an initial Compton interaction in the liquid xenon volume and it is therefore directly comparable with the efficiency of a conventional double scatter telescope. The energy of 
the events in the sample was required to be totally contained. Additionally, the scattered $\gamma$-ray was required to travel a distance of at least $5.4 \mathrm{~cm}$ to insure good angular resolution. Since $5.4 \mathrm{~cm}$ corresponds roughly to the average photon attenuation length for few $\mathrm{MeV} \gamma$-rays, only $37 \%$ of all events are accepted. Despite this stringent cut a significant improvement in efficiency over that of a conventional telescope is realized.

By relaxing the restriction on the average distance between the two interaction points, a substantial fraction of the rejected events can be recovered, at the expense of the angular resolution. Since this cut is implemented during the off-line data analysis, it can be easily adjusted to maximize the information on the physical quantities of interest. The most obvious enhancement in detection efficiency is achieved by including the events which start with pair production. The second curve in Fig. 8, through the points marked as $\square$, shows in fact the overall efficiency of the telescope, with these events included. The requirement of full energy containment is still applied.

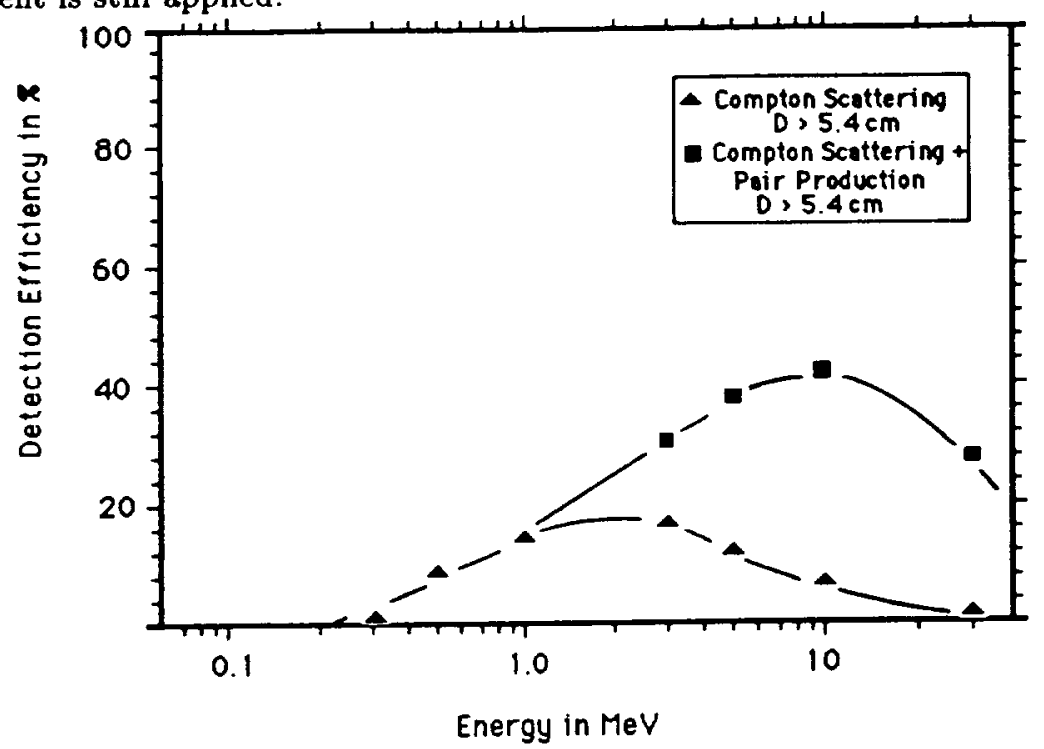

Figure 8. Detection efficiency of the proposed liquid xenon chamber for $\gamma$-ray events with an initial Compton interaction $(\boldsymbol{\Lambda})$ and for those including an initial pair production ( $\boldsymbol{(}$ ) at various $\gamma$-ray energies.

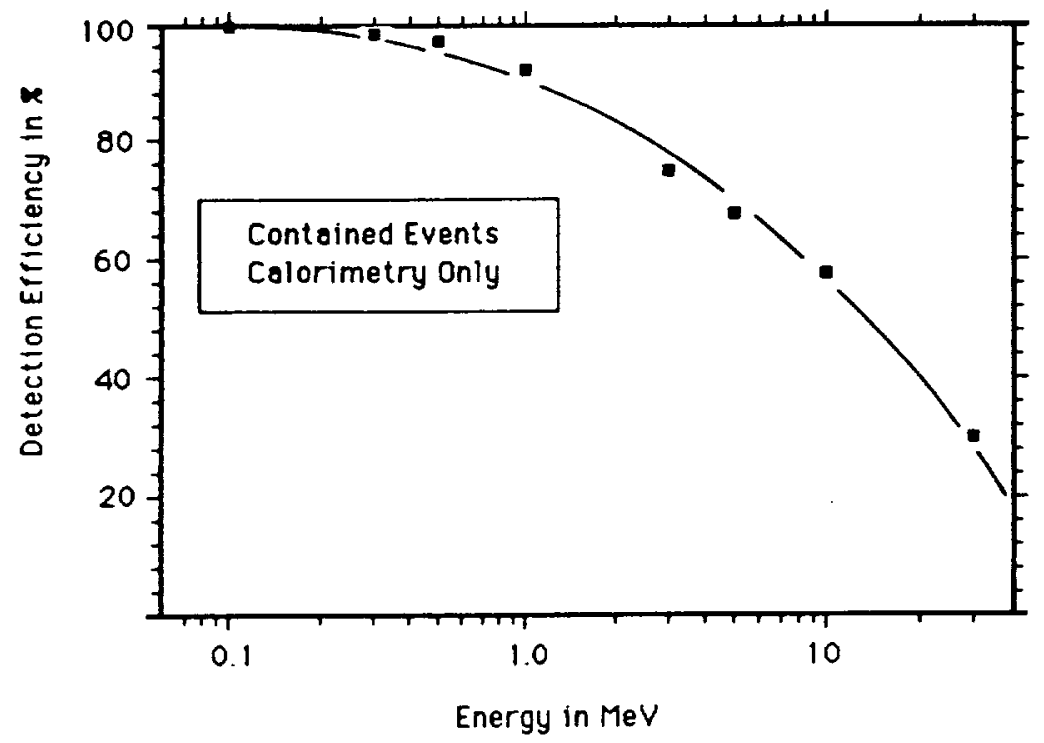

Figure 9. Detection efficiency of the proposed liquid xenon chamber as a high resolution $\gamma$-ray spectrometer at various $\gamma$-ray energies. 
At lower energies most $\gamma$-rays interact via photo-effect and the incoming direction cannot be reconstructed. However, the energy of these events is still measured and the liquid xenon chamber then operates as a high resolution $\gamma$-ray spectrometer. Since the only condition is that the total energy is contained within the volume, the detector efficiency is very high, as shown in Fig. 9.

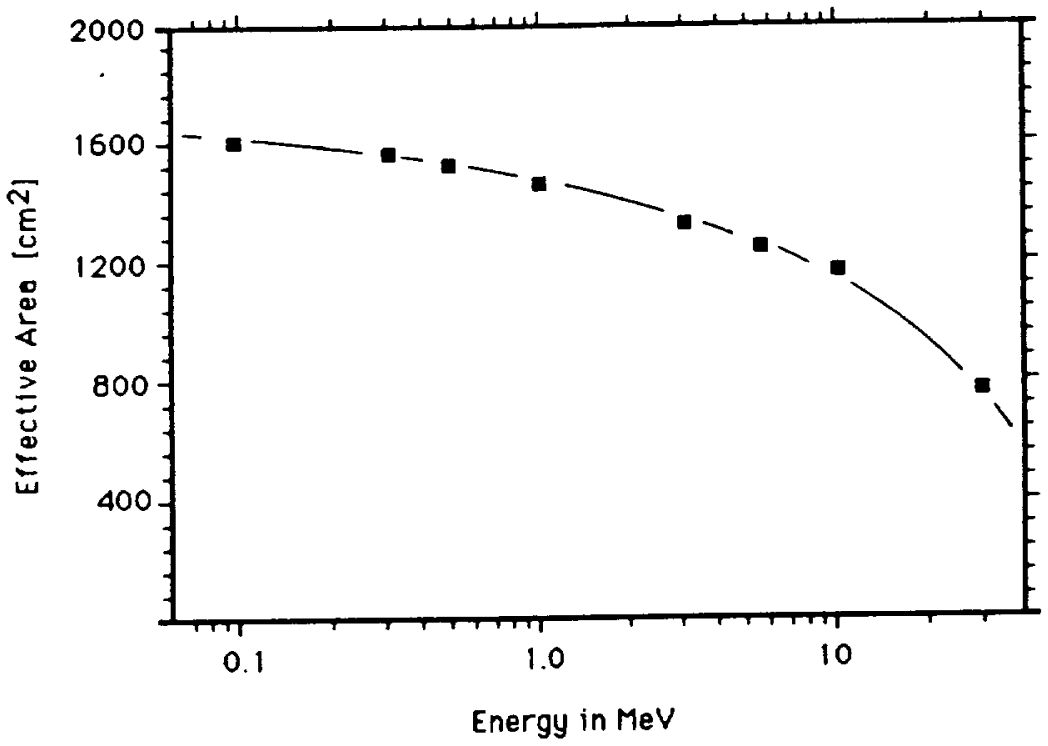

Figure 10. Effective detection area of the proposed liquid xenon imaging chamber at various $\gamma$-ray energies.

The detection efficiency of Fig. 8 and 9 was calculated assuming vertical $\gamma$-ray incidence in the center of a liquid xenon detector with a geometrical area of $1600 \mathrm{~cm}^{2}$. Only small changes in the overall detection efficiency are expected for non-vertical incidence. For $\gamma$-rays entering the chamber very close to the boundary, the efficiency will be reduced as part of the interaction products might leave the active volume. One can calculate the effective detection area for Compton and pair production events, by taking these effects into account. Figure 10 gives the Monte Carlo result for the effective area at various $\gamma$-ray energies.

\section{SUMMARY}

We have discussed the application of a liquid xenon Time Projection Chamber as a high resolution imaging telescope for $\gamma$-ray energies between 1 and $30 \mathrm{MeV}$. The combination of excellent energy and position resolution, imaging capability with a large field of view, high efficiency and low background, make it an ideal instrument for sensitive observations of a wide class of astrophysical phenomena emitting low and medium energy $\gamma$-rays.

This work was supported by DARPA (N00014-86C-0086) and partially by NASA grant NAGW-1370.

\section{REFERENCES}

E. Aprile, W. Ku , J. Park and H. Schwartz Nucl. Instr. and Meth., A261, 519 (1987).

E. Aprile, W. Ku and J. Park, IEEE Trans. Nucl. Sci., 35(1), 37 (1988).

E. Aprile and M. Suzuki, IEEE Trans. Nucl. Sci., 36(1), 311 (1989).

K. Deiters, et al., Nucl. Instr. and Meth., 180, 45 (1981).

T. Doke, et al., Nucl. Inst. and Meth., 134, 353 (1976).

R.L. Ford and W.R. Nelson, the EGS Code System (Version 4), SLAC-210, (1978).

S. Kubota, et al., Phys. Rev., B20, 3486 (1979) and Nucl. Instr. and Meth., 196, 101 (1982).

S. Kubota, et al., Phys. Rev., B21, 2632 (1980).

H.J. Mahler, et al., IEEE Trans. Nucl. Sci., NS-30, 86 (1983).

L.S. Miller, et al., Phys. Rev., 166, 871 (1968). 
R. Muller, et al., Phys. Rev. Lett., 27, 532 (1971).

E. Shibamura, et al., Phys. Rev., A20, 2547 (1979). 



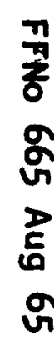

207

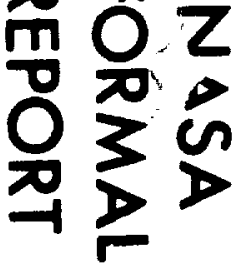

